\title{
El uso del rumor en la prensa digital de videojuegos. Análisis de contenido de los medios especializados: Hobby Consolas, Vandal y 3DJuegos
}

The use of rumor in digital press of video game. Analysis of contents of the specialized media: Hobby Consolas, Vandal and 3DJuegos

Guillermo Paredes-Otero, Facultad de Comunicación

Calle Américo Vespucio, 27, 41092 Sevilla

guillermoparedes87@gmail.com | Orcid: http://orcid.org/0000-0002-9732-521X

DOI: http://dx.doi.org/10.12795/Ambitos.2019.i45.09

\begin{abstract}
Resumen
La cultura del videojuego ha provocado que el ocio interactivo sea la industria de entretenimiento audiovisual más lucrativa en España. Su implantación en numerosas áreas de la sociedad y su consideración más allá de una actividad lúdica ha derivado en el aumento del interés informativo por este fenómeno no solamente en la prensa especializada sino en los medios de comunicación generalistas. Esto nos lleva a dos preguntas: ¿cómo se informa sobre los videojuegos? ¿Se contrasta la información para difundir mensajes verificados como indican los principios del periodismo? Partiendo de la base de que esta especialización periodística no es ajena a la
\end{abstract}


desinformación presente en ciertas publicaciones emitidas por los medios de comunicación y con estas cuestiones como punto de partida, se pretende estudiar el uso del rumor en el periodismo de ocio interactivo. Para ello se apuesta por un análisis de contenido de este tipo de publicaciones de tres medios especializados y referentes del sector, Hobby Consolas, Vandal y 3DJuegos, durante el periodo de un año y atendiendo a la frecuencia de publicación, aspectos gramaticales, las fuentes informativas y las temáticas. Los resultados del análisis han permitido esbozar un perfil de cómo es el rumor en la prensa de videojuegos y demostrado que el uso de este tipo de informaciones es una práctica perfectamente implementada en la práctica periodística y común para comunicar las novedades de futuros lanzamientos y crear expectación entre los usuarios.

\begin{abstract}
Video game culture has made interactive entertainment the most lucrative audiovisual entertainment industry in Spain. Its implementation in many areas of society and its consideration beyond a playful activity has resulted in the increase of informative interest by this phenomenon not only in the specialized press, but also in the generalist media. This bring us to two questions: how is it reported about video games? Is the information contrasted to spread verified messages as the principles of journalism indicate? Starting from the basis that this journalistic specialization is not strange to the misinformation present in certain publications issued by the media and with these questions as a starting point, we intend to study the use of rumor in interactive leisure journalism. For this purpose, we are committed to an analysis of the content of this type of publications by three specialized media and industry benchmarks, Hobby Consolas, Vandal and 3DJuegos, during the period of one year, and taking into account the frequency of publication, grammatical aspects, information sources and the themes. The results of the analysis have allowed us to outline a profile of what the rumor is in the videogame press and demonstrated that the use of this type of information is a practice perfectly implemented in journalistic and common practice to communicate the news of future releases and create expectation among the users.
\end{abstract}

Palabras clave: Información, prensa digital, prensa especializada, rumor, videojuegos.

Keywords: Information, digital press, specialized press, rumor, video games.

\title{
1. INTRODUCCIÓN
}

El papel de los videojuegos en la sociedad ha evolucionado desde su concepción a mediados de los años 70 pasando en apenas cincuenta años de ser simplemente "multimedia interactivo" (Levis, 1997, p. 36), "juegos electrónicos" (Law, 2016, p. 7) o "software de entretenimiento" (Frasca, 2001, p. 4) a "artefactos culturales que funcionan en base a reglas que permiten la manipulación lúdica de entornos virtuales 
por parte del usuario, entendidos como entornos distintos a los de la vida cotidiana" (Anyó, 2016, p. 14) e incluso un fenómeno cultural basado en un producto digital que requiere de la interactividad del usuario para su funcionamiento y con una trascendencia más allá de la esfera del entretenimiento. Ejemplo de esta evolución es que el ocio interactivo ha dejado el ámbito exclusivo del entretenimiento para introducirse en otras áreas de la sociedad como la educativa -con el término gamificación- o su profesionalización hasta el punto de ser considerado una carrera universitaria e incluso posibilidad laboral como demuestran los eSports o deporte electrónico.

Tal relevancia explica que el ocio interactivo sea a día de hoy el sector audiovisual más lucrativo en España, llegando a facturar la cantidad de 1.359 millones de euros en 2017, una cantidad que duplica a la facturada por el cine y es seis veces mayor que la música en dicho periodo (AEVI, 2018). Si a esto le sumamos que España es el cuarto país europeo en consumo de videojuegos y está dentro del TOP 10 en el ranking mundial, se puede llegar a entender que haya un interés periodístico en el tema hasta el punto de hablar de una especialización periodística.

El presente trabajo pretende rellenar un vacío científico existente en cuanto a periodismo especializado en videojuegos se refiere, prestando atención en concreto a cómo los medios digitales relacionados con esta temática utilizan el rumor, un tipo de texto tan presente en la actividad periodística -incluso en especialidades como el periodismo deportivo- y relacionado con términos tan habituales en la actualidad como la desinformación o las fake news. Por tanto consideramos relevante conocer cómo tiene cabida el rumor a la hora de informar sobre el sector audiovisual más lucrativo en España.

\section{LA PRENSA ESPECIALIZADA EN VIDEOJUEGOS}

Al mismo tiempo que el videojuego ha evolucionado, su tratamiento informativo ha cambiado de igual forma por parte de la prensa especializada. Simplemente hay que observar dicho periodismo especializado en España. Las primeras publicaciones, como fueron ZX o MSX Club, contenían páginas completas de códigos para que los usuarios pudieran programar sus propios juegos (Martínez, 2016). Estas revistas especializadas en videojuegos nacieron en los años 80 por el interés de los usuarios en la información relacionada con los juegos que llegaban al mercado español (Muñoz y Sebastián, 2010), publicaciones que, independientemente de la editorial, tenían una misma finalidad:

El objetivo original de estas publicaciones era el de funcionar como guía de compras, no sólo informando de todos los lanzamientos, sino comentando de manera crítica su contenido y, en definitiva, asesorando a los lectores sobre los 
juegos que merecía la pena comprar y los que no (p. 219).

Este propósito se sigue produciendo en la actualidad, independientemente de si son medios de comunicación en papel o digitales, pero al mismo tiempo se compagina con otro tipo de informaciones más allá de la actualidad del desarrollo de videojuegos y su posterior análisis como forma de orientar su posible adquisición por parte de los usuarios.

En los últimos años, está cobrando especial relevancia también toda la actualidad relativa a la repercusión cultural del videojuego y su consideración como forma de expresión artística a través de informaciones que versan sobre exposiciones y eventos, libros que se publican sobre esta temática, artistas que incluyen referencias extraídas del mundo del ocio electrónico en sus obras o la relación del videojuego con otras artes (López-Redondo, 2017, p. 372).

Con las nuevas tecnologías los medios especializados en videojuegos han sufrido una revolución a la hora de informar sobre el tema ya que como indican Muñoz y Sebastián (2010):

Las revistas virtuales y sitios webs dedicados a los videojuegos (desde las grandes webs a los humildes blogs) han experimentado una interesante evolución en los últimos años, pasando de ser simples revistas adaptadas a Internet a incluir herramientas que las han convertido en verdaderas comunidades virtuales (p. 222).

Solamente hay que ver las webs de los medios especializados en videojuegos más visitadas en España -Hobby Consolas, Meristation, Vandal, IGN España o 3DJuegos(Martínez, 2016, p. 161) para comprobar que todas han dado el salto a las redes sociales y tienen sus propios perfiles en al menos Facebook, Twitter y YouTube. Al mismo tiempo sus webs se aprovechan de las características innatas del periodismo online como la hipertextualidad (Meso, 1993), la interactividad (Diaz Noci, 2002) y el uso de contenidos multimedia (Salaverría, 2005).

Sin embargo, el periodista de videojuegos se ve sujeto a otra de las características innatas de los medios digitales que en muchos casos impide la correcta labor periodística. Es el caso de la inmediatez o la necesidad de publicar cuanto antes la información en la web hace que, como señala Diaz Noci (2002), en muchos casos se carezca de tiempo para contrastar la información o analizar su veracidad. Durandin (1995) coincide en que "en ausencia del tiempo necesario para verificarlas, las noticias se propagan, con independencia de su verdad o falsedad, sin saber demasiado de dónde ni de quién emanan" (p. 193). López-Redondo (2017), por su parte, añade una segunda dificultad en el periodismo de videojuegos: 
La distancia geográfica y el hermetismo con el que estas compañías llevan a cabo sus proyectos hacen que el flujo de información entre ellas y los medios sea casi unidireccional y el periodista tenga que conformarse, en la mayoría de los casos, con publicar únicamente la los datos que recibe a través de notas de prensa (p. 373).

\section{LA ERA DE LA DESINFORMACIÓN}

La prensa de videojuegos tampoco escapa de la desinformación, entendida esta como una forma definir algunas prácticas del actual panorama informativo, "un sistema mediático de fabricación de mentiras" (Serrano, 2009, p. 12) hasta el punto de que lo que ofrecen los medios "no es buen contenido informativo, ellos venden audiencias, nos venden a nosotros a las agencias de publicidad" (p. 31). Kapuscinski (2001) explica esto afirmando que "desde que está considerada como una mercancía, la información ha dejado de verse sometida a los criterios tradicionales de la verificación, la autenticidad o el error. Ahora se rige por las leyes del mercado" ${ }^{1}$.

Autores como Fraguas ya calificaban en 1985 la desinformación como una forma de oposición "en todos los textos periodísticos y literarios a información en el sentido amplio" (p. 10) así como una forma que en vez de disminuir el coeficiente de incertidumbre del sujeto, aumentarlo en beneficio de aquel que niega a admitir la necesidad de la doble libertad de informar y de informarse" (p. 160). Por su parte para Durandín (1995) "la desinformación sería la cara negativa de la información (...), proponemos definirla como un conjunto organizado de engaños en una era en la que los medios de comunicación se hallan enormemente desarrollados" (pp. 24-25).

Mas de Xaxàs va un paso más allá y define esta era como una "dictadura de la información" en la que "todo ha de ser inmediato y, a ser posible, live, vivo y fresco, encerado como una manzana de colmado caro. Las noticias deben ser tentadoras, casi subversivas, porque nuestra capacidad de atención se reduce" (2005, p. 25). Galdón (1994) añade un elemento más a la desinformación como es la sustitución de la calidad por la cantidad con el peligro que esto conlleva, y expresa su razón de ser:

No conduce a una sociedad informada ya que lo que en realidad aumenta es la 'información innecesaria', la no dirigida, la no significativa. Lo que en realidad se produce es una acumulación de hechos sin sentido incapaz de proporcionar una imagen coherente, fidedigna de la realidad (pp. 30-31).

Dentro de esta esfera de desinformación, el papel del periodista se reduce al de "una pieza idónea para narrar, de forma natural, una historia con una clara intención publicitaria. Las noticias informativas, pensadas y escritas sólo por su interés general y social, pierden terreno frente al periodismo publicitario" (Mas de Xaxàs, 2005, p. 34). Al mismo tiempo los medios de comunicación omiten en el proceso de su labor 
periodística "la verificación crítica (...) de las afirmaciones realizadas por los actores sociales a poderes públicos. Se impide así diferenciar la verdad de la falsedad" (Galdón, 1994, p. 44). De esta forma la función actual de los medios, como indica Serrano (2009) "no es informar sino desinformar, no es fomentar el análisis y el debate democrático, sino diluirlo y silenciarlo" (p. 73).

En una era de desinformación donde está tan de moda las fake news, entendidas como "informaciones falsas diseñadas para hacerse pasar por noticias con el objetivo de difundir un engaño o una desinformación deliberada para obtener un fin político o financiero" (Amorós, 2018, p. 35), para esta investigación hemos decidido centrarnos en otra herramienta de desinformación como es el rumor, de hecho hay autores como Kapferer (1989) que lo califican como el medio más antiguo del mundo por su naturaleza de "vehículo de las noticias" ( $p$. 11) y definida como "la aparición y circulación en la colectividad social de informaciones que aún no han sido confirmadas públicamente por las fuentes oficiales, o que éstas han desmentido" (p. 24).

Otras definiciones también coinciden en la naturaleza no verificada de la información. Allport y Postman (1947) explican el rumor como "una proposición específica (o tópica) para la creencia, que se transmite de persona a persona, generalmente de forma oral, sin estándares seguros de evidencia presentes" (p. 9). Baumeister y Vohs (2007), por su parte, los califica como "noticias, excepto que las noticias van acompañadas de evidencia sólida" (p. 771).

Kapferer (1989) ante esta falta de verificación aclara que "el rumor no es por la fuerza 'falso'. Pero es por la fuerza no oficial" (p. 273), una información paralela y alejada de cualquier control que "pone en duda a las autoridades, planteando 'quién tiene derecho a hablar y de qué'. Como información paralela y a veces opuesta a la información oficial, el rumor constituye un poder alternativo" (p. 24).

Aunque los rumores y las fake news puedan parecer lo mismo y así lo atestigüen autores como Amorós (2018) lo cierto es que ambas técnicas de desinformación son diferentes. Por un lado, mientras el rumor es una información cuya veracidad es dudosa o no puede corroborarse por las fuentes oficiales e implicadas, las fake news, como su nombre indica, son noticias directamente falsas. Cosa que no ocurre con el rumor, el cual deja un atisbo de duda y la posibilidad de que los datos que ofrecen sean verdaderos.

Si se presta atención a la finalidad con la que se difunden, los rumores, aunque pretendan en ocasiones "calumniar, desvalorización del sistema de información del adversario y la puesta en circulación de noticias contradictorias" (Durandín, 1995, pp. 198-199), cuando se trata del periodismo de videojuegos encontramos un nuevo objetivo: el de informar para elevar el interés del usuario por un juego y crear una 
disposición de comprar, es decir, se lanzan rumores en el periodismo especializado en videojuegos con fines comerciales.

Las fuentes, es decir el origen de la información que posteriormente se va a difundir, apuntan a ser el aspecto fundamental para diferenciar el rumor de las fake news. López-Redondo (2017) refleja que a la hora de informar sobre ocio interactivo hay dos tipos de fuentes:

Las propias compañías y determinadas webs especializadas localizadas en tres grandes focos informativos a nivel mundial: Japón, Reino Unido y Estados Unidos. Estos tres países son los principales mercados de videojuegos del mundo, donde esta industria tiene un mayor arraigo, y donde se genera el mayor volumen de información en torno al ocio electrónico. La mayor parte de la información que se publica cada día depende, por tanto, de los anuncios y comunicados oficiales o convocatorias de prensa de las empresas de videojuegos (p. 370).

A lo largo de la exposición de los resultados de esta investigación comprobaremos cómo el origen de los rumores provienen de más de dos tipos de fuentes y que en ningún caso son oficiales, al contrario que ocurre con el resto de informaciones sobre videojuegos. Al mismo tiempo, el uso de términos como 'fuente anónima' o 'fuentes cercanas a...', más allá de preservar oculta la identidad de quienes suministran la información, se utiliza como recurso para desinformar. Las fuentes anónimas se usan "para disfrazar lo que ha sido imposible corroborar. Sirven para hacer realidad nuestras intuiciones $y$, junto con su compañero de cama, el rumor, son consecuencia de la cobardía, avaricia, delación, maldad, egolatría, vulgaridad y falsedad del periodismo" (Mas de Xaxàs, 2005, p. 129).

En cualquier caso, una posible explicación ante la presencia de tantos rumores en las webs de videojuegos españolas la ofrece López-Redondo (2017) localizándola en las políticas de comunicación que llevan a cabo las empresas de ocio interactivo, la cual dificulta la labor del periodista hasta el punto de no ser capaz éste de poder contrastar algunas informaciones. Sin embargo, esto no parece afectar a quienes llegan a publicar los rumores debido a que la consideran:

Información que no perjudica a nadie y que simplemente sirve para alimentar las expectativas de los usuarios que son, al fin y al cabo, consumidores de un producto de entretenimiento. En el origen de muchos de estos rumores están las propias compañías que, a través de una compleja estrategia de marketing viral, pretenden contribuir a generar mayor expectación en torno a un determinado videojuego (p. 373). 


\section{EL RUMOR EN LOS MEDIOS DIGITALES DE VIDEOJUEGOS}

Antes de pasar a explicar los objetivos de esta investigación así como la metodología llevada a cabo para su obtención consideramos pertinente detenernos en los propios medios cuyas publicaciones se van a tener en cuenta en este estudio, prestando especial atención a su actividad periodística y en un primer acercamiento al uso del rumor dentro de sus publicaciones. Estos medios se han escogido al ser tres de los medios de comunicación más antiguos y visitados que actualmente existen en España.

\subsection{Hobby Consolas}

Hobby Consolas nació originariamente en formato papel en junio de 1991 y tiene el honor de ser la revista de videojuegos española más longeva, todavía publicándose de forma mensual. Su fundación corrió a cargo de Hobby Press, quienes pertenecen actualmente a Axel Springer, encargados de su edición.

La revista dio el salto al formato web en 2010 bajo el nombre de Hobby News (hobbynews.es) como el portal de videojuegos de Axel Springer. Dos años más tarde, el 1 de agosto de 2012, se convertiría en HobbyConsolas.com, con David Martínez y Daniel Quesada como web managers de las secciones Juegos y Entretenimiento respectivamente.

La web se centra en las noticias, análisis y reportajes de los videojuegos independientemente de la plataforma a la que pertenezcan. Cuenta además con Entretenimiento una sección dedicada a contenidos más allá del ocio interactivo como críticas a películas, series o cómics, y con Hobby Industrias dedicada al sector industrial del videojuego en España y a la formación.

Hobby Consolas es desde febrero de $2018^{2}$ el canal con el que el grupo 20minutos informa de videojuegos después de que Vandal rompiera la colaboración dos meses antes para unirse a El Español.

\subsection{Vandal}

Esta web nació el 29 de junio de 2005 y se definen a sí mismos como espacio online pionero en tratar la actualidad informativa de las videoconsolas, cuyo origen se remonta al verano de 1997. El éxito de sus publicaciones hicieron que el medio pasara a ser parte del grupo de comunicación 20minutos en 2010 para convertirse en su canal de videojuegos. Acuerdo que duró hasta diciembre de 2017 cuando la web firmó un acuerdo con El Español, de Pedro J. Ramírez, para convertirse este último en el principal inversor y accionista del portal de ocio interactivo ${ }^{3}$. 
Vandal fue la primera web especializada en videojuegos en ofrecer vídeos en Full HD y en cubrir el evento de videojuegos E3 en Los Ángeles. Al margen de informar de la actualidad de los videojuegos con noticias, reportajes, análisis y avances, la web incluye guías y foros. Vandal también le presta especial atención a los eSports, a la creación de vídeos y las informaciones audiovisuales y cuenta con la sección Vandal Random enfocada al cine y a la televisión.

\subsection{DJuegos}

Esta web nació el 29 de junio de 2005 y se definen a sí mismos, según indica su web, como

Una revista online especializada en videojuegos y líder de audiencia en España. Un equipo de periodistas y profesionales a los que ante todo les encanta disfrutar con los juegos. Un medio independiente donde tu opinión es muy importante y además, cuenta de verdad. Una revista de videojuegos abierta de par en par a ideas nuevas y a todo tipo de jugadores. Una comunidad que se construye en equipo y que gira alrededor del videojuego, la cultura del videojuego.

Actualmente la web pertenece a Webedia, grupo internacional ligado al entretenimiento y al ocio y que cuenta entre sus filas con espacios online de este sector como Sensacine o IGN.

A diferencia de Hobby Consolas y Vandal, 3DJuegos se dedica únicamente a informar de la actualidad del ocio interactivo, dejando a un lado los espacios de cine y televisión. Por otro lado, cuenta también con su propia sección dedicada al deporte electrónico (eSports). Como puede verse, estamos hablando de tres medios de comunicación pertenecientes a grupos empresariales y con experiencia de décadas a la hora de informar sobre ocio interactivo.

Atendiendo a los textos considerados como rumores que publican dichas webs y como paso previo a la investigación realizada, para comprobar el calado que tiene este tipo de publicaciones dentro de la labor periodística especializada se ha producido al introducir el término 'rumor' dentro de los buscadores de los tres medios dando los siguientes resultados. 3DJuegos es el medio que más referencias a rumores tiene con 642 resultados en su hemeroteca digital. Le sigue Hobby Consolas con 516 textos relacionados con esta temática desde que se fundó su web en 2010. Por su parte el término 'rumor' aparece en los titulares de 265 noticias publicadas por Vandal. Como puede verse, el uso de rumores en estos medios no son una práctica casual sino que es habitual recurrir a este tipo de publicaciones para informar sobre ocio interactivo. 
Teniendo estos últimos datos en cuenta, en esta investigación nos hemos planteado como objetivo principal analizar cómo los medios digitales y españoles especializados en videojuegos usan el rumor en el ejercicio periodístico. Al mismo tiempo hay marcado como objetivo más específico el esbozar un perfil de la publicación típica cuando se trata de usar el rumor atendiendo a su temática, contenido y aspectos lingüísticos.

Estos objetivos propuestos junto a la metodología que a continuación se detallará, al igual que la muestra de análisis, tienen como finalidad el poder responder a la siguiente pregunta de investigación: ¿Cómo los medios digitales españoles y especializados en videojuegos usan el rumor a la hora de informar sobre ocio interactivo?

\section{METODOLOGÍA}

Para la consecución de los objetivos planteados y la respuesta a la pregunta de investigación propuesta se ha apostado por la realización de un análisis de contenido que permita conocer cómo los medios digitales, españoles y especializados en ocio interactivo usan el rumor durante la realización de la labor periodística.

Buscando la correcta aplicación de este método, se han consultado estudios previos sobre periodismo especializado en ocio interactivo como el trabajo realizado por López-Redondo (2012), enfocado al tratamiento informativo del videojuego tanto en la prensa generalista como en los medios especializados. Al mismo tiempo, se ha tenido en consideración el trabajo de doctorado de Nogales-Bocio (2013) por el uso de dicha técnica de investigación en el estudio periodístico.

Determinado el método y consultados antecedentes de su uso, el análisis de la presente investigación se ha realizado sobre una muestra de 197 textos periodísticos los cuales debían de cumplir con unas características concretas:

- Los textos debían pertenecer a Hobby Consolas, Vandal y 3DJuegos, tres de los medios especializados en videojuegos más visitados en España.

- Las publicaciones tenían que haber sido subidas a las distintas webs entre octubre de 2017 y septiembre de 2018. Consideramos que un año es un periodo temporal lo suficientemente amplio como para comprobar el uso que realizan estos medios del rumor y esbozar un perfil fidedigno del texto sobre videojuegos típico basado en rumores.

- Por último, se han seleccionado aquellos textos periodísticos que, dentro de dicho periodo temporal, tenía el término 'rumor' en el título. 
Obtenida la muestra, se ha prestado atención a unos aspectos muy concretos, los cuales consideramos necesarios y fundamentales para la obtención de los objetivos propuestos. Las categorías en las que se ha dividido la ficha de este análisis de contenido son las siguientes:

- Medio: sirve para identificar los distintos documentos y contabilizar la cantidad de publicaciones basadas en rumores que publica cada web especializada en ocio interactivo de la muestra.

- Título: en esta categoría se pretende comprobar el uso que el medio le da a la hora de utilizar el término 'rumor' dentro del título de la información.

- Fecha de publicación: la función de esta categoría es saber en qué períodos del año hay mayor y menor cantidad de publicaciones basadas en rumores con el objetivo de hacer un perfil más completo.

- Temática principal: la idea es comprobar sobre qué temas se basan los rumores que publica la prensa especializada en videojuegos.

- Fuente: esta categoría servirá para conocer de dónde provienen los rumores que, sin tener confirmación oficial en ninguno de los casos, los medios digitales y especializados en ocio interactivo acaban publicando en sus webs y ofreciendo a sus usuarios.

- Tiempos verbales: su finalidad consiste en comprobar qué expresiones gramaticales se usan para informar sobre actualidad en ocio interactivo en base a rumores.

\section{RESULTADOS OBTENIDOS}

\subsection{Títulos}

Atendiendo a cómo los medios especializados en videojuegos que componen la muestra utilizan el término 'rumor' en sus títulos podemos afirmar que existen tres formas estrategias de uso: en primer lugar la palabra 'rumor' es una más dentro del título como demuestran los ejemplos 'Crecen los rumores acerca de una colaboración entre For Honor y Prince of Persia' o 'Rumores sobre el desarrollo de Devil May Cry 5 y Soul Calibur VI'.

Otra práctica empleada consiste en destacar de alguna forma la palabra 'rumor', ya sea incluyendo el término entre paréntesis -'Microsoft anunciaría hoy la rebaja en el precio de Xbox One X (rumor)'- o bien separándolo a través de pausas a la hora de leer el título -'PS5 utilizará un procesador Ryzen de última generación, según un rumor'-. Por último, la última estrategia consiste en hacer del rumor un tipo de texto 
propio. Al igual que los reportajes o las entrevistas, se ha comprobado la existencia de ejemplos cuyos titulares comienzan indicando el tipo de texto en qué consiste -'Rumor: Nintendo emitirá un Direct de Switch Online la madrugada del día 7'-.

\subsection{Fecha de publicación}

En función de la periodicidad a la hora de publicar rumores sobre videojuegos a lo largo del año analizado, se ha podido comprobar cómo el período en el que menos noticias de este tipo se difunden en las webs especializadas es en el último trimestre del año, entre los meses de octubre y diciembre, con apenas 15 rumores de un total de 197 textos. Los meses de verano, concretamente julio y agosto también presentan un número reducido de rumores -21 en concreto- coincidiendo con el periodo vacacional y en el que la actividad laboral desciende.

Aunque como muestra la siguiente gráfica, el número de rumores tiene una curva descendente durante el primer cuatrimestre del año, la media es superior a los dos periodos citados anteriormente, viéndose una media de 21 rumores mensuales entre enero y abril. Sin embargo, el mes que más rumores se publican, como se puede comprobar, es mayo con 37 textos periodísticos cuya veracidad no está comprobada, una cantidad muy superior a la vista en el resto de meses del año analizado. De hecho hay días en los que un mismo medio llega a publicar varios rumores, por ejemplo el 30 de mayo de 2018 en los que Vandal llega a difundir hasta cuatro rumores diferentes.

Una posible explicación de por qué hay tantos rumores en mayo está en que durante el mes de junio tiene lugar el E3 en Los Ángeles, considerada como la mayor feria de los videojuegos y en la que las compañías más prestigiosas del sector, como Sony, Nintendo o Microsoft, presentan sus futuros juegos en desarrollo. Por lo tanto se empieza a especular con qué juegos o novedades se mostrarán en dicha convención. De hecho Hobby Consolas llega a encontrar en esto la justificación a la difusión de rumores a lo largo de dicho mes con expresiones como 'Con el E3 2018 a tan solo un mes de distancia, es normal que empiecen a surgir nuevos rumores sobre algunos de los proyectos más interesantes de la industria' o 'no parece muy descabellado si tenemos en cuenta la proximidad del E3 2018'.

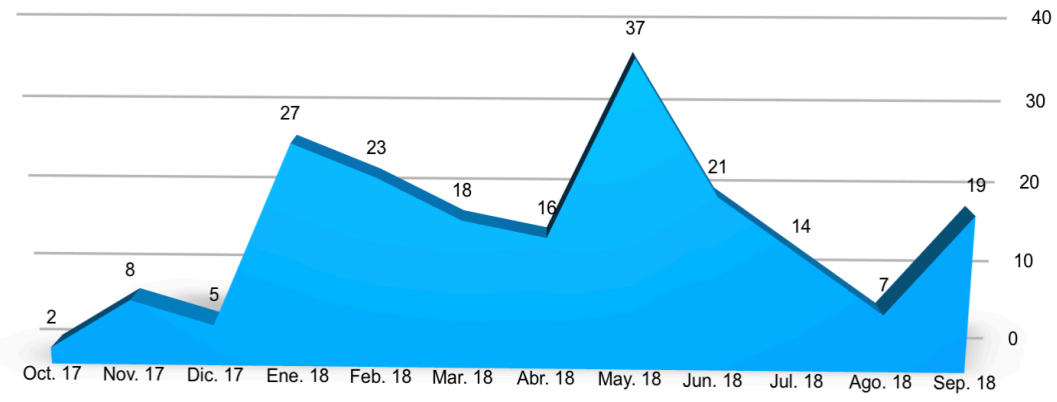

Ámbitos. Revista Internacional de Comunicación | ISSN: 1139-1979 | E-ISSN: 1988-5733, №. 45. (2019) 


\section{Gráfica 1. Número de rumores según el mes del año \\ Fuente: Elaboración propia}

También llega a ser recurrente en los medios analizados la mención del E3 con enunciados tipo 'tendremos que estar muy atentos a todas las conferencias del E3 2018', 'Por ahora, se tratan de declaraciones confusas que sin duda se esclarecerán el próximo mes durante la celebración del E3', 'Todas estas incógnitas podrían ser resueltas finalmente durante la conferencia de Xbox en el E3 2018'. Expresiones que, por otro lado, parecen justificar que, como el E3 va a tener lugar pronto, no hace falta corroborar la información ya que ya se verá durante el evento si resultan ser verdad o no los datos publicados.

Cabe destacar y centrándonos en los medios analizados en concreto como Vandal es el medio que más rumores publica con 113 textos, es decir, prácticamente un rumor cada 3,23 días. Le sigue Hobby Consolas con 67 rumores, uno cada 5,44 días, y muy lejos de los 17 textos basados en rumores de 3DJuegos, dicho de otra forma, este medio difunde un rumor cada 21,47 días.

\subsection{Temática principal}

Respecto al tema que abordan los textos, más de la mitad de la muestra analizada trata sobre novedades de juegos ya confirmados dentro de los planes de las compañías de ocio interactivo (37\%) -'Medievil Remastered incluiría los dos Medievil, según un rumor' o 'Rumor: GTA 6 transcurre en Miami y Latinoamérica'- y sobre el desarrollo de nuevos títulos de ocio interactivo (24\%) -'El próximo Assassin's Creed podría apodarse Dynasty, según un rumor' o 'Cobra fuerza el rumor sobre el regreso de Spyro the Dragon'-.

Un $14 \%$ de los textos analizados corresponden a 'Futuros Lanzamientos', categoría que se diferencia de la anterior en que los textos versan sobre juegos que ya están confirmados su desarrollo e incluso ya han salido a la venta y la novedad radica en que se va a lanzar en nuevos dispositivos o plataformas, como es el caso de la noticia 'Rumor: Dragon Ball FighterZ y Fortnite se anunciarán para Switch en el E3'. Diferente al tema de 'Desarrollo de videojuegos' que se centra en juegos cuyo lanzamiento aún no se ha producido. 

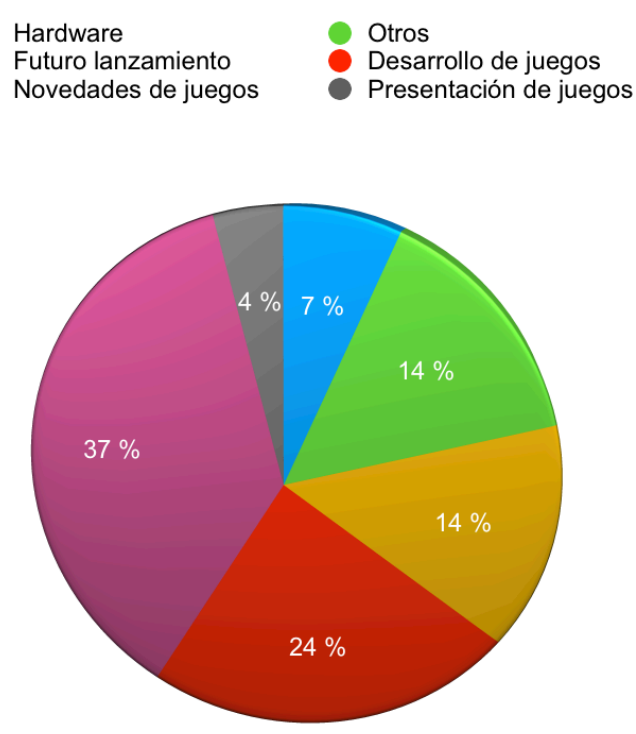

Gráfica 2. Temáticas de los rumores analizados

Fuente: Elaboración propia

Por otro lado, un $7 \%$ de la muestra se centra en el hardware, es decir, complementos y aspectos técnicos de las consolas y soportes de ocio interactivo -'Rumor: PlayStation 5 usaría una CPU de AMD de la gama Ryzen'- mientras que un $4 \%$ de las noticias son sobre supuestas presentaciones oficiales de videojuegos -Rumor: Pokémon para Switch se presentaría este mes'-.

Finalmente se ha comprobado cómo hasta un $14 \%$ de la muestra analizada son noticias que no podemos añadir a las temáticas anteriores y se han agrupado en el tema de 'Otros'. Estamos hablando de textos relacionados con nuevas opciones de jugabilidad -'Rumor: PlayStation Now podría incorporar una opción de descarga'-, actualidad sobre las empresas del sector -'Rumor: Demandan a Warner por copiar contenidos de Fallout Shelter en Westworld'- o eventos de las compañías de videojuegos -'Un rumor sugiere un Nintendo Direct este mes con grandes anuncios'-.

\subsection{Fuentes}

Este apartado se centra en el aspecto más importante de la investigación: las fuentes que toman de referencia los medios de comunicación españoles y especializados en videojuegos para informar en sus webs de la actualidad del ocio interactivo. Se busca saber en base a qué o quién obtienen los datos para luego publicarlos en forma de información. 
Lo primero que debe tenerse en cuenta es que en ninguna de las informaciones provienen de fuentes propias de los tres medios que se analizan en esta investigación. Dicho de otra forma los datos que publican no los han obtenido por sí mismos de las fuentes primarias sino que escriben textos en base a otras publicaciones. Por lo tanto, a la hora de informar sobre actualidad en videojuegos en base a rumores, podemos decir que los medios especializados españoles se dedican a reproducir los datos que han conseguido otros, por lo que no es información original.

En segundo lugar todas las fuentes que citan los medios analizados provienen del extranjero, es decir, son internacionales, salvo un caso cuyo origen era la Revista Oficial de Nintendo en España. Profundizando en cómo estas fuentes han adquirido la información sobre la que se informa hemos podido constatar cómo, dejando a un lado que en ningún caso hay confirmación oficial ya que si la hubiera no estaríamos hablando de rumores, es habitual la expresión 'fuentes cercanas a...', sin llegar a mencionar cuales son realmente esas fuentes, como si con usar dicha frase ya estuviera la información contrastada y verificada. Esta falta de verificación se extrapola a la actividad de los tres medios analizados, los cuales simplemente optan por hacerse eco de los datos que han obtenido otros, sin molestarse en contrastarlos.

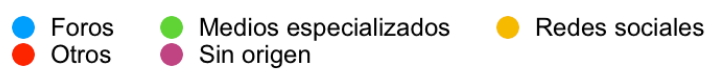

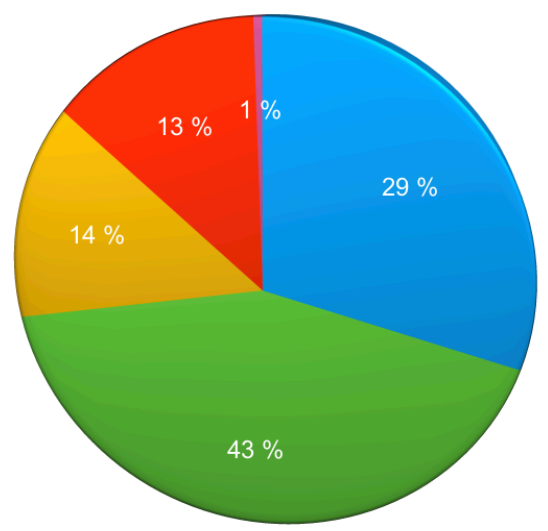

Gráfica 3. Fuentes del origen de los rumores analizados

Fuente: Elaboración propia

Atendiendo a las fuentes que citan Hobby Consolas, Vandal y 3DJuegos en las noticias consideradas como rumores, el principal lugar desde donde obtienen la información es de webs especializadas en ocio interactivo. El $43 \%$ de los textos analizados tienen como fuente los datos obtenidos por sitios online que informan sobre videojuegos, como PC Gamer, Nintendo Life, Go Nintendo, Kotaku o VG247, así como por aquellas filtraciones obtenidas por periodistas especializados. Por otra parte un 
$29 \%$, es decir más de una cuarta parte de la muestra, tiene una información proveniente de foros como ResetEra, Reedit o 4chan y de los comentarios de sus usuarios.

Llama la atención cómo, teniendo en cuenta la importancia que tienen en la actualidad a nivel informativo, las redes sociales sean la tercera vía más utilizada desde la que obtener información para publicar rumores. Un 14\% de la muestra analizada basa su información en las publicaciones vistas en Twitter y YouTube.

Por otro lado, un $13 \%$ de los textos analizados tienen como fuente principal aquellas con un origen que no se puede agrupar en los casos anteriores como es la imagen de una camiseta en la web de Funko, catálogo de Mediamarkt, un supuesto cartel visto en una tienda japonesa e incluso una felicitación navideña de los desarrolladores de un juego. Algo tan simple como los ejemplos recién citados basta para componer alrededor un entramado de datos y hacerlo pasar por actualidad informativa en ocio interactivo.

Finalmente, un $1 \%$ de las noticias que componen la muestra carecen del origen que da pie al rumor, es decir, no presentan la fuente en el texto.

\subsection{Tiempos verbales}

El análisis lingüístico realizado a las noticias que componen la muestra se ha centrado en observar los tiempos verbales que se emplean para informar sobre la actualidad del ocio interactivo en base a rumores. De los 197 textos analizados el tiempo verbal predominante es el condicional, el cual aparece hasta en 165 documentos. Son frecuentes las expresiones tipo 'sería', 'rondaría', 'se realizaría', 'podría llegar', 'estaría pensando', 'se retrasaría' o 'podría estar en desarrollo' que indican la inseguridad propia del tiempo condicional. Es decir, queda reflejado que los datos que forman estos textos periodísticos no es segura, no está confirmada por fuentes oficiales.

A pesar de esta falta de verificación por los datos que se publican, los medios especializados analizados hacen uso de otros tiempos verbales que sí expresan seguridad como son el presente y el futuro. Por un lado, en 91 noticias se usan verbos conjugados en presente como 'está trabajando', 'señala', 'está haciendo pruebas' o 'está actualmente en desarrollo'. El futuro, en cambio, es usado para ofrecer los datos con rotundidad, a pesar de que no haya confirmación oficial, a través de enunciados como 'volverá', 'será presentada', 'se lanzará', 'buscará replicar el éxito' o 'permitirá explorar'. Esto ocurre en 76 de los textos analizados.

Por último, las conjugaciones verbales en pasado también tienen cabida en los textos periodísticos basados en rumores, siendo frecuentes las declaraciones que comienzan por 'han sugerido', 'especulaba', 'comenzó a publicar', 'ha filtrado', 'ha anunciado', 'han 
revelado'. En definitiva, expresiones que suelen enfocarse en cómo el usuario o medio que publica primero el rumor ha obtenido los datos más que en los datos en sí mismos.

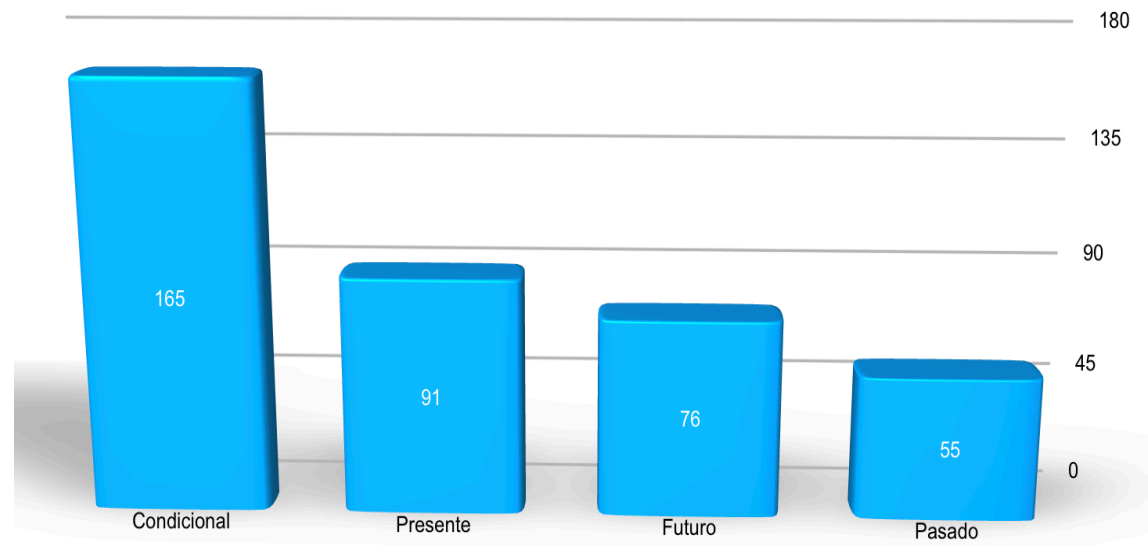

Gráfica 4. Tiempos verbales usados en los rumores

Fuente: Elaboración propia

\subsection{Otras observaciones}

A lo largo del análisis de la muestra seleccionada se ha podido comprobar algunas otras acciones en los textos, las cuales en ningún caso son hechos aislados. Por ejemplo, en un $22,61 \%$ de los casos el propio medio pide cautela a la hora de tomar en serio la información de la noticia mientras que en un $26,90 \%$ se destaca que no hay confirmación oficial por parte de las empresas a las que se hace alusión, proviniendo la información, como se ha visto, de fuentes secundarias.

A pesar de no haber confirmación oficial en ninguno de los casos, el medio en cuestión ofrece motivos para creer en el rumor en un $22,61 \%$ de los textos analizados. Para finalizar, se ha visto como en un $22,33 \%$ de la muestra la web en cuestión abandona la actitud neutral de simplemente informar sobre el rumor que ha surgido para optar por una postura activa y contribuye al mismo con opiniones o especulando sobre la información.

\section{CONCLUSIONES}

El uso del rumor está perfectamente implementado en la actividad periodística de los medios especializados en videojuegos analizados llegando a ser un tipo de publicación recurrente para informar sobre la actualidad del ocio interactivo. Concretamente, las informaciones sin contrastar ni verificar que guardan relación con novedades de 
juegos cuyo lanzamiento ya está confirmado así como sobre el desarrollo de nuevos títulos son las temáticas más habituales. Para informar sobre estos temas las webs españolas de videojuegos recurren principalmente a medios y periodistas especializados de carácter internacional y a foros centrados principalmente en comentarios de los propios usuarios. En ningún caso los rumores provienen de fuentes propias cuyo origen no pueden mencionar.

De esta forma, llama la atención el predominio de fuentes extranjeras como origen del rumor en todos los textos analizados. La inminencia del evento de videojuegos E3 de Los Ángeles hace que mayo sea el mes donde mayor cantidad de textos basados en rumores se publican, por encima de la cifra media habitual del resto del año. En contrapartida, el último trimestre del año y los meses de verano son las fechas con menor presencia de rumores.

Atendiendo a cuestiones lingüísticas, para ofrecer los datos basados en fuentes no confirmadas destaca el uso predominante de tiempos verbales en condicional, lo cual ofrece la inseguridad propia de no tener los datos verificados. Esto, sin embargo, contrasta con el hecho de que se emplea verbos en presente y futuro cuya impresión es la opuesta, es decir, que los datos que se ofrecen son verídicos. Aspectos como la titulación de las piezas informativas, los tiempos verbales y, sobre todo, las temáticas que se abordan, hacen que la finalidad a la hora de publicar rumores respondan a la necesidad de crear una expectación sobre el producto o videojuego en cuestión.

Finalmente, la respuesta a la pregunta con la que partía esta investigación es que los medios digitales y españoles especializados en videojuegos usan el rumor a través de noticias con las que informar sobre novedades y futuros desarrollos de juegos empleando para ello fuentes provenientes de otros medios especializados y foros de usuarios. Para ello utilizan principalmente el uso de modos verbales en condicional y presente $y$, aunque resaltan que no hay confirmación oficial, contribuyen especulando con nueva información.

\section{Notas}

${ }^{1}$ La cita pertenece a un discurso pronunciado por Ryszard Kapuscinski durante una ceremonia de entrega de premios de periodismo en Estocolmo, posterior-mente publicado en Le Monde Diplomatique, julio-agosto de 1999. En el apartado de referencias aparece el enlace web desde el que se ha obtenido dicha cita.

2 Información obtenida de la noticia publicada en la web de 20 Minutos titulada' '20minutos' y Hobby Consolas se unen para producir contenido de videojuegos' el 6 de febrero de 2018. El texto completo se puede consultar en el siguiente enlace: https://bit.ly/2Q6QhXr

${ }^{3}$ El Español lo anunciaba a través de la noticia 'EL ESPAÑOL se convierte en primer inversor de Vandal para hacerlo líder en videojuegos y entretenimiento', la cual se puede consultar en https://bit.ly/2AC6jDn. 


\section{Referencias}

20 Minutos. (6 de febrero de 2018). '20minutos' y Hobby Consolas se unen para producir contenido de videojuegos. 20 Minutos. Recuperado de https://bit.ly/2Q6QhXr.

AEVI (2018). Anuario 2017. Anuario de la Industria del Videojuego. Recuperado de https://bit.ly/2sK1fud.

Allport, G.W. y Postman, L. (1947). The psychology of rumor. Recuperado de https://bit.ly/2lyCJTI.

Amorós, M. (2018). Fake News. La verdad de las noticias falsas. Barcelona: Plataforma Editorial.

Baumeister, R. F. y Vohs, K. D. (2007). Encyclopedia of social psychology. Recuperado de https://bit.ly/2Im9Ef5.

Díaz Noci, J. (2002). La escritura digital: hipertexto y construcción del discurso informativo en el periodismo electrónico. Bilbao: Universidad del País Vasco.

Durandín, G. (1995). La información, la desinformación y la realidad. Barcelona: Paidós.

El Español (15 de diciembre de 2017). EL ESPAÑOL se convierte en primer inversor de Vandal para hacerlo líder en videojuegos y entretenimiento. El Español. Recuperado de https://bit.ly/2AC6jDn.

Frasca, G. (2001). Videogames of the opressed: videogames as a means for critical thinking and debate (Tesis Doctoral). Recuperado de https://goo.gl/skvbep.

Fraguas de Pablo, M. (1985). Teoría de la desinformación. Madrid: Alhambra.

Galdón, G. (1994). Desinformación. Método, Aspectos y Soluciones. Navarra: Eunsa.

Kapuscinski, R. (2001). ¿Reflejan los media la realidad del mundo? Rebelión. Recuperado de https://bit.ly/2Rfd2sg.

Kapferer, J.N. (1989). Rumores. El medio de difusión más antiguo del mundo. Barcelona: Plaza \& Janes.

Law, Y.Y. (2016). The travelling gamer: an ethnography of video game events (Tesis doctoral). Recuperado de https://bit.ly/2lz1YW3.

Levis, D. (1997). Los videojuegos, un fenómeno de masas. Qué impacto produce sobre la infancia y la juventud la industria más próspera del sistema audiovisual. Barcelona: Paidós. 
López-Redondo, I. (2012). El tratamiento del videojuego: de la prensa generalista a las revistas especializadas. Análisis comparativo de las ediciones impresas y digitales de El País, El Mundo, Público y 20 Minutos (Tesis doctoral). Recuperado de https://bit.ly/2KDbhXA.

López-Redondo, I. (2017). El rumor como noticia en la prensa del video-juego. Estudio comparativo de dos revistas digitales: Meristation y Vandal. En A. Larrondo, K. Meso y S. Peña (Eds.), Actas del $9^{\circ}$ Congreso Internacional de Ciberperiodismo (pp. 360-373). Bilbao: Universidad del País Vasco.

Martínez, D. (2016). De Microhobby a Youtube. La prensa de videojuegos en España. Palma de Mallorca: Dolmen Editorial.

Mas de Xaxàs, X. (2005). Mentiras. Viaje de un periodista a la desinformación. Barcelona: Ediciones Destino.

Meso, K. (1993). Introducción al ciberperiodismo. Breve acercamiento al estudio del Periodismo en Internet. Bilbao: Universidad del País Vasco.

Muñoz, D. y Sebastián, A. (2010). "La relación de los videojuegos con los medios de comunicación", en Carrillo, J. y Sebastián, A. (coords), Marketing Hero. Las herramientas comerciales de los videojuegos, (pp. 201-231. Madrid: Esic.

Nogales, A.I. (2013). Metodología empírico-periodística del análisis de contenido y su aplicación. Aproximación estructural a la cobertura de los atentados del 11-M a través de El País, El Mundo, Abc y La Razón (Tesis Doctoral). Recuperado de https://bit.ly/2EX9RV9.

Salaverría, R. (2005). Redacción periodística en internet. Navarra: EUNSA.

Serrano, P. (2009). Desinformación. Cómo los medios ocultan el mundo. Barcelona: Península. 